\title{
Selection criteria for high-dose-rate surface brachytherapy and electron beam therapy in cutaneous oncology
}

\author{
Ivan M. Buzurovic, PhD, Desmond A. O’Farrell, MS, Thomas C. Harris, MS, Scott Friesen, MS, Martin T. King, MD, \\ Robert A. Cormack, PhD, Phillip M. Devlin, MD \\ Department of Radiation Oncology, Dana Farber/Brigham and Women's Cancer Center, Harvard Medical School, Boston, MA, USA
}

\begin{abstract}
Purpose: High-dose-rate (HDR) brachytherapy is an alternative treatment to electron external beam radiation therapy (EBRT) of superficial skin lesions. The purpose of this study was to establish the selection criteria for HDR brachytherapy technique (HDR-BT) and EBRT in cutaneous oncology for various clinical scenarios.

Material and methods: The study consists of two parts: a) EBRT and HDR-BT treatment plans comparison analyzing clinical target volumes (CTVs) with different geometries, field sizes, and topologies, and b) development of a prediction model capable of characterization of dose distributions in HDR surface brachytherapy for various geometries of treatment sites.

Results: A loss of CTV coverage for the electron plans $\left(D_{90}, D_{95}\right)$ was recorded up to $45 \%$, when curvature of the applicator increased over $30^{\circ}$. Values for $\mathrm{D} 2 \mathrm{~cm}^{3}$ for both plans were comparable, and they were in range of $\pm 8 \%$ of prescription dose. An increase in higher doses $\left(\mathrm{D} 0.5 \mathrm{~cm}^{3}\right.$ and D0.1 $\left.\mathrm{cm}^{3}\right)$ was observed in HDR-BT plans, and it was greater for larger lesions. The average increase was $3.8 \%$ for $\mathrm{D} 0.5 \mathrm{~cm}^{3}$ and $12.3 \%$ for $\mathrm{D} 0.1 \mathrm{~cm}^{3}$. When CTV was approximately flat, electron plans were comparable with HDR-BT plans, having lower average D2 $\mathrm{cm}^{3}, \mathrm{D} 0.5 \mathrm{~cm}^{3}$, and D0.1 $\mathrm{cm}^{3}$ of $7.7 \%$. Degradation of quality of electron plans was found to be more dependent on target curvature than on CTV size.

Conclusions: Both EBRT and HDR-BT could be used in treatments of superficial lesions. HDR-BT revealed superior CTV coverage when the surface was very large, complex, curvy, or rounded, and when the topology was complicated. The prediction model can be used for an approximate calculation and quick assessment of radiation dose to organs-at-risk (OARs), at a depth or at a lateral distance from CTV.
\end{abstract}

J Contemp Brachytherapy 2021; 13, 2: 195-204 DOI: https://doi.org/10.5114/jcb.2021.105288

Key words: high-dose-rate brachytherapy, electron therapy, dose distributions, prediction model.

\section{Purpose}

Recently, relevant literature on radiation oncology suggested that there was no alternative treatment to electron beam therapy [1]. We have recently witnessed a rapid growth and development of different treatment modalities and techniques in radiation therapy, with the purpose of improving the quality of cancer treatments. When treating shallow tumors, radiation beams that pass through the entire patient are not favorable. The electron external beam radiation therapy (EBRT) was proven to be suitable for shallow tumors at a certain depth (rarely deeper than a cm). The principal applications of EBRT are: a) treatment of skin and lip cancers, b) chest wall irradiation for breast cancer, c) administering a boost dose to lymph nodes, and d) treatments of the head and neck [2]. Dose distributions and dose characterizations in EBRT are well documented
[2-5]. Percent depth doses for various electron beams [4] show that a certain dose level can be deposited into deeper-seated tissue due to electron penetration, generation of secondary electrons, and photon contamination. This characteristic of electron beams is not desirable in cases where radiation dose needs to be deposited at a very shallow depth under the skin surface, e.g., less than $5 \mathrm{~mm}$. In such cases, surface brachytherapy can be good or even better treatment modality for skin treatments [6].

Aside from EBRT, two additional advanced radiation therapy treatment techniques for malignant and non-malignant diseases of the skin include high-dose-rate brachytherapy (HDR-BT) and electronic brachytherapy [6, 7]. Adapted for a specific disease and topography, these brachytherapy techniques are used for effective, accurate, and safe delivery of radiation. Moreover, the complexity of clinical target volumes (CTVs), e.g., curvy, rounded, or 
targets with complicated topology, is in a direct relationship with the adequate choice of treatment modality used in skin treatments. The prescription dose for some cutaneous diseases (cutaneous $\mathrm{T}$ cell and B cell lymphoma, Merkel cell carcinoma, basal cell carcinoma, squamous cell carcinoma, rare adnexal neoplasms: eccrine, apocrine, sebaceous, and hair follicle tumors, and other rare cutaneous cancers) has to be delivered at shallow depths; thus, superficial HDR-BT is an alternative to EBRT.

As noted, requirements for dose distributions in skin treatments are related to desirable dose depositions at certain depths with organs at risk (OARs) sparing beyond that depth. A primary characteristic of HDR-BT is the rapid and sharp dose fall-off, with the possibility to spare critical organs near treated regions or clinical targets. Clinicians often need to choose between EBRT and HDR-BT for the treatments of skin targets. Clinical decisions are usually made based on previous experience and availability of resources. However, critical decision-making conditions that need to be considered are treatment site geometry, position, size, and topography of the target. These components are directly related to favorable dose distributions in both EBRT and HDR-BT. When the skin ought to be treated, various target geometries require proper adaptation of the flap (or custom-made) applicators or the electron field to this treatment site. Consequently, the dose at depth on central axis (CAX) and field edges changes with the variation of curvatures and the size of applicators.

The purpose of this study was to investigate the influence of such parameters on the quality of treatment plans generated under the same conditions (identical computed tomography (CT) images and the same patients), using EBRT and HDR-BT for skin treatments. EBRT and HDR-BT treatment plans were compared to identify which treatment modality resulted in more favorable dosimetry. Target coverage and dose distributions were evaluated under various clinical scenarios, such as large targets, small targets, curvy surfaces, rounded surfaces, and the like. All generated HDR-BT treatment plans were clinically delivered; however, it was investigated whether EBRT resulted in improved radiation dose distributions in various cases. Additional purpose of this study was to generate robust quantitative criteria that could result in a set of straightforward recommendations for either treatment modality prior to delivery. These decision criteria were based on the knowledge of geometry of clinical targets, including size, anatomic curvatures, and topography that could be obtained by visual inspection of the target or evaluation of the clinical images, such as CT. As a result, OARs' doses could be predicted based on the presented charts; thus, either EBRT or HDR-BT could be recommended based on particular circumstances.

\section{Material and methods}

The first part of this study included generation, evaluation, and data analysis of EBRT and HDR-BT treatment plans using CT images for 37 treatment sites to aid in developing a relevant conclusion regarding treatment modalities. The second part of this study involved characterizing the dose distributions of HDR-BT in skin. The goal was to generate a predictive dose distribution model of various CTV shapes and sizes. These dose distribution models were designed to be a counterpart to the well-known electron dose distribution parameters, such as $R_{\mathrm{p}}, R_{50}$, and depths of $80 \%$ and $90 \%$ isodose lines, respectively.

\section{Patients and clinical targets}

The study was performed with a cohort of 37 patients who had been diagnosed with cutaneous $\mathrm{T}$ cell or $\mathrm{B}$ cell lymphoma or angiosarcoma, and had been treated within a five-month period. The targets were present on the preauricular cheek (2), thighs (6), abdominal wall (4), forearm (6), flank and hip (8), scalp (6), and the whole face (5). The size of CTV varied from $4 \times 6 \mathrm{~cm}^{2}$ to $23 \times 40 \mathrm{~cm}^{2}$. The treatment sites, target sizes, and depths are presented in Table 1. One of the most relevant factors for dose distribution evaluation was the target curvature. For this study, the curvature was defined as the distance along CAX (CAX distance) from the peripheral margin to the plane perpendicular to the point of incidence (vertex) of CAX. The topology of targets varied from almost flat to parabolic, with a minimum and maximum CAX distance of $4.7 \mathrm{~mm}$ and $70 \mathrm{~mm}$, respectively. Additionally, characteristics of the clinical targets summarized in Table 1 showed wide variations in size, geometry, and topology. These variations were crucial for the EBRT and HDR-BT plans comparisons.

\section{Treatment planning}

First, the EBRT and HDR-BT plans were generated. The HDR-BT plans were used for patient treatments,

Table 1. Treatment sites, applicator/CTVs size, and CAX distance of CTVs for all patients

\begin{tabular}{|c|c|c|c|c|c|c|}
\hline \multirow[t]{2}{*}{ Group } & \multirow{2}{*}{$\begin{array}{l}\text { Number } \\
\text { of patients }\end{array}$} & \multirow[t]{2}{*}{ Site } & \multicolumn{2}{|c|}{ Applicator/CTVs size } & \multicolumn{2}{|c|}{ CAX distance } \\
\hline & & & $\operatorname{Min}\left(\mathrm{cm}^{2}\right)$ & $\operatorname{Max}\left(\mathrm{cm}^{2}\right)$ & $\operatorname{Min}(\mathrm{cm})$ & $\operatorname{Max}(\mathrm{cm})$ \\
\hline 1 & 2 & $\begin{array}{l}\text { Preauricular } \\
\text { cheek }\end{array}$ & $4 \times 6$ & $6 \times 7$ & 0.00 & 0.47 \\
\hline 2 & 6 & Thighs & $10 \times 10$ & $36 \times 23$ & 0.40 & 4.50 \\
\hline 3 & 4 & Abdominal wall & $14 \times 20$ & $26 \times 23$ & 0.80 & 3.80 \\
\hline 4 & 6 & Forearm & $10 \times 12$ & $27 \times 23$ & 0.20 & 7.00 \\
\hline 5 & 8 & Flank and hip & $6 \times 8$ & $40 \times 23$ & 0.52 & 4.80 \\
\hline 6 & 6 & Scalp & $12 \times 18$ & $18 \times 20$ & 5.20 & 6.97 \\
\hline 7 & 5 & Whole face & 47 catheters & 66 catheters & - & - \\
\hline
\end{tabular}




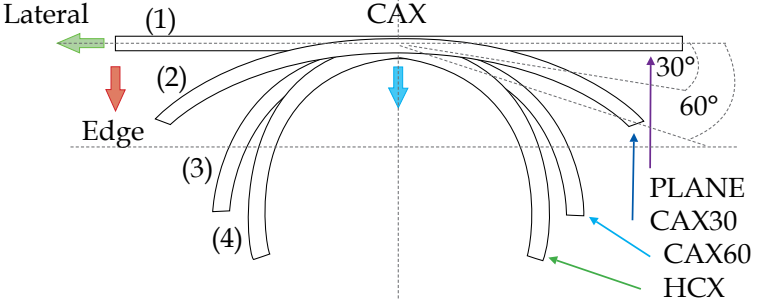

Fig. 1. The following applicators were designed to create dose distributions for the most common geometries: planar (PLANE) with no curvature, curved to $30^{\circ}$, and $60^{\circ}$ with respect to the CAX (CAX30 and CAX60), hyperbolic arc with central angle of $215^{\circ}$ (HCX)

while the EBRT plans were used for comparisons only. All patients underwent a simulation using CT prior to planning, so the EBRT and HDR-BT treatment plans were generated on identical CT images. Specific details on treatment planning for both techniques are presented later in this section. To analyze the data obtained from dosimetric plans, we compared dosimetric parameters including the coverage of CTV $\left(\mathrm{D}_{100}, \mathrm{D}_{95}, \mathrm{D}_{90}, \mathrm{D}_{50}\right)$, maximum plan doses (D2 $\left.\mathrm{cm}^{3}, \mathrm{D} 0.5 \mathrm{~cm}^{3}, \mathrm{D} 0.1 \mathrm{~cm}^{3}\right)$, surface doses, and irradiated volumes. The CTVs for HDR-BT plans were created by adding margins to visible gross tumor volumes. These margins were in the range of $2-5 \mathrm{~cm}$. The EBRT plans were generated by adding an additional margin to encompass possible patient movement and setup uncertainties.

In the second part of this study, the dose distribution for a total of 9 HDR-BT cases was created for CTVs of $10 \times 10 \mathrm{~cm}^{2}$ and $20 \times 20 \mathrm{~cm}^{2}$, with a step size of $1 \mathrm{~cm}$ and a prescription depth of $3 \mathrm{~mm}$, to generate a predictive model of dose distributions in surface brachytherapy. The geometry of applicators was planar with no curvature (PLANE), curved to $30^{\circ}$ and $60^{\circ}$ with respect to CAX (CAX30 and CAX60), and half-cylinder (HCX). These treatment plans were used to emulate typical treatments of the skin on a patient's abdomen, flank, arm, forearm, neck, breast scar, chest wall, etc. The geometry of targets is presented in Figure 1. The dose fall-off was recorded in three regions: a) on the CAX of applicator, b) at the edge of applicator on the axis parallel to CAX (CAXE), and c) at the edge of applicator on the axis perpendicular to CAX (CAXL), as shown in Figure 1. This choice was made to be able to evaluate and predict dose distributions at depth (case $a$ and b) and lateral spread of the dose with respect to the surface applicator or custom mold.

An additional dose distribution was created, in which the applicators formed a dome shape (DS) with a diameter of $16 \mathrm{~cm}$ (Figure 2). This treatment plan was used to emulate the treatment of an average-sized scalp. All treatment plans were optimized to deliver a prescription dose at $8 \mathrm{~mm}$ equidistantly from parallel planes including dwell positions. This optimization was equivalent to the clinical arrangement since the source-to-surface distance for the flap applicators was $5 \mathrm{~mm}$ and the prescription depth was $3 \mathrm{~mm}$ in most of the clinical cases.

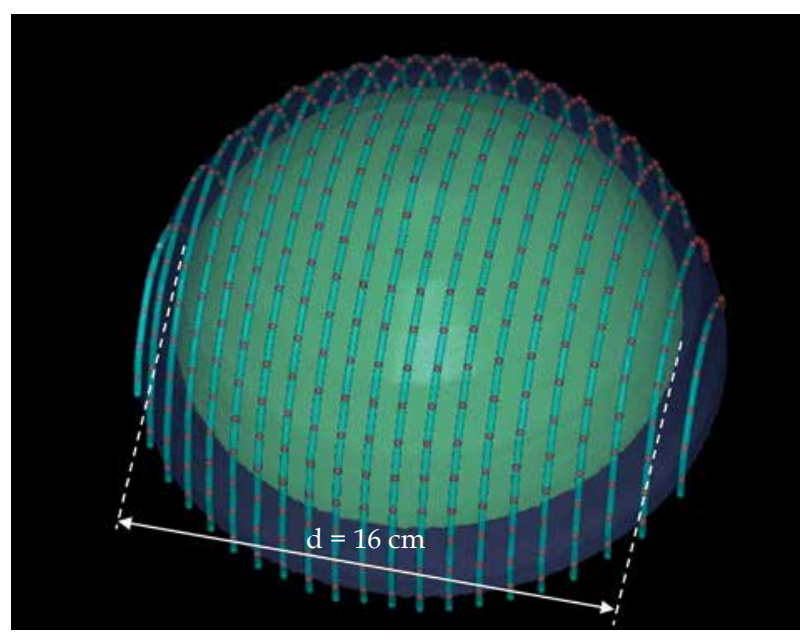

Fig. 2. A dome-shaped (DS) model with a diameter of $16 \mathrm{~cm}$ was utilized to create dose distributions for scalp treatments. This is the typical clinical case with patients diagnosed with angiosarcoma of the scalp

\section{HDR treatment planning}

The HDR planning was performed using Oncentra Brachy planning system (Elekta Brachytherapy version 4.5.3, Veenendaal, The Netherlands). The HDR-BT treatment planning method was clinically proven to be efficacious and effective [6]. The general planning details were summarized: a) in most cases, it was not necessary to contour the clinical target area (equivalent to the CTV); b) there was no need to contour the skin, either; c) skin markers (wires) delineated the CTV; they were crucial for planning and they had to be contoured in planning images; d) $100 \%$ of the prescription dose was $3 \mathrm{~mm}$ under the skin (to reach the dermis) or deeper up to $8 \mathrm{~mm}$ to reach the depth of the target. It was possible to generate the plan with a non-constant depth, i.e., the depth of central part of the target could be up to $8 \mathrm{~mm}$ in depth, whereas the depth at the target periphery could be $3 \mathrm{~mm}$; e) the $125 \%$ isodose line stayed off or just touched healthy skin (OAR) (to spare stem cells and vasculature). In rare situations, the skin dose can be up to $135 \%$ of prescription dose for a treatment depth of $3 \mathrm{~mm}$. Commercial flap applicators and custom-made applicators were used for HDR treatments. The prescription dose used in these treatments complied with published guidelines [8] for both palliative and definitive treatments.

The catheters were reconstructed either from the tip or connector end, maintaining consistent approach within each plan. Additional planes (paraxial, parasagittal, and paracoronal) were used for accurate catheter reconstruction to detect as many catheters as possible on a single slice. This technique was particularly used for catheter reconstructions in large CTVs with more than 30 catheters per plan. Only the dwell position inside or at the margin of CTV was activated to obtain adequate coverage. The treatment plans were initially normalized with respect to multiple dose points $3 \mathrm{~mm}$ into the target, to span the dose distribution evenly. Further optimization of the isodose lines fine-tuned the prescription dose. The process started with global optimization and ended 
with local adjustments of the isodose lines. Special attention was paid to dose distribution at the edges of applicators, so that local high-dose regions and poor coverage of targets could be avoided.

\section{EBRT treatment planning}

The EBRT planning was performed using EMC-10028 Electron Monte Carlo planning module of Eclipse (Varian Medical Systems, Inc., Palo Alto, USA). Single and matched beam techniques were used. The electron plans were generated using $6 \mathrm{MeV}$ energy and $1 \mathrm{~cm}$ bolus to achieve an optimal dose distribution at the prescription depth. The electron beams were set perpendicular to the largest possible surface area.

\section{Treatment plan validation}

All brachytherapy treatment plans were used in clinical treatments; they were evaluated and approved by an attending physician. All electron treatment plans were reviewed by an attending physician and approved to be used for treatment plan comparison.

\section{Results}

The results of the study were presented in two major sections: the first, compares the dosimetric effects of EBRT and HDR-BT plans over a clinically relevant range of topographies, and the second, investigates the predictive capability for OARs metrics from an HDR-BT plan at varying levels of depth and laterality.

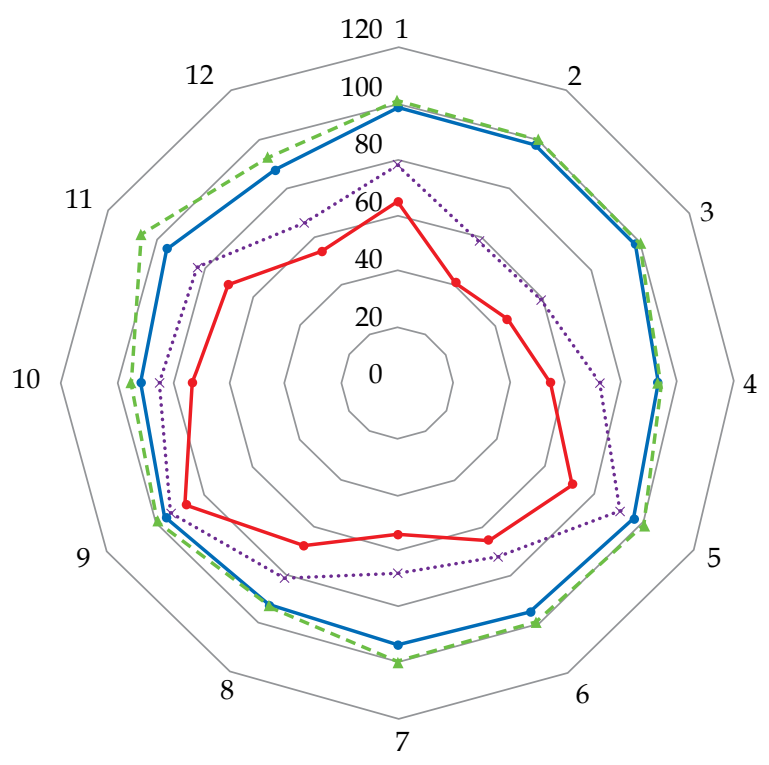

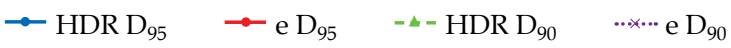

Fig. 3. Significant loss of the coverage for the electron plans was noticed as the applicator angle increased over $30^{\circ}$ (purple and red lines). The green and blue lines represent the coverage for the HDR-BT plans, indicating that HDR-BT can adapt the dose to complicated topologies. The graph presents $D_{90}$ and $D_{95}$ data from 12 representative cases

\section{HDR vs. EBRT: plan comparisons}

The significant degradation of CTV coverage for electron plans $\left(D_{90}\right)$ was recorded as $41.5 \%$ to $20 \%$ when the anterior-posterior CAX distance increased from $24 \mathrm{~mm}$ to $58 \mathrm{~mm}$ (as the curvature increased). Therefore, a decrease of CTV coverage for the electron plans was noted when the applicator formed an angle greater than $30^{\circ}$, according to the notation from Figure 1. When the CAX distance was increased further, the loss of CTV coverage remained nearly constant. The $\mathrm{D}_{50}$ for both HDR-BT and the electron plans was similar. The $\mathrm{D}_{95}$ and $\mathrm{D}_{100}$ degraded similarly as the $\mathrm{D}_{90}$ did for the electron plans as the applicator angle increased (Figure 3). The values for D2 $\mathrm{cm}^{3}$ for both plans were comparable and were in the range of $-8 \%$ to $7.5 \%$ of the prescription dose. An increase in higher doses (D0.5 $\mathrm{cm}^{3}$ and D0.1 $\mathrm{cm}^{3}$ ) was noted in HDR-BT plans, and it was even greater for larger targets. The average increase was $4 \%$ for D0.5 $\mathrm{cm}^{3}$ and $12 \%$ for D $0.1 \mathrm{~cm}^{3}$. When the CTV was approximately flat (that is, the CAX distance was less than $10 \mathrm{~mm}$ ), the electron plans were comparable to the HDR plans, having a lower D2 $\mathrm{cm}^{3}$, $D 0.5 \mathrm{~cm}^{3}$, and D0.1 $\mathrm{cm}^{3}$ of $8 \%$ on average. It was observed that surface dose $(\mathrm{d}=0)$ was lower for the electron plans in the range of $6-20 \%$ of the prescription dose. However, this advantage cannot be used to improve the loss of CTV coverage for the electron plans, because whenever the surface dose increased to the predefined limits, the prescription dose was delivered deeper than desired. Irradiated volumes for the electron plans were up to two times greater if the plans were normalized, to obtain an adequate CTV coverage. In general, the degradation of quality of the electron plans was found to be more dependent on the target curvature than on the CTV size. Figure 4 shows two representative cases planned with EBRT and HDR-BT. It was possible to achieve equivalent coverage and dose distributions in case 1 (small and almost flat surface) with both modalities; however, this was not feasible with a large CTV with curvy geometry (complex topology). The electron plans for the whole face and scalp treatments (11 patients in total) were suboptimal, and their statistical data were excluded from the numerical comparative analysis. In such cases (Figure 5), clinically acceptable plans were generated only with HDR-BT; no substitution with EBRT planning was feasible.

\section{Dose distribution in HDR skin brachytherapy}

In this section, a dose distribution prediction model was presented using relevant distributions (Table 2) for various applicator shapes and sizes as shown in Figures 1 and 2. Corresponding figures (Figures S1-S9) were presented in the Supplement section of this paper. Isodose distributions were calculated using TG-43 formalism, and they were confirmed with Monte Carlo calculations. Issues related to the presence of bone and lungs in surface brachytherapy [9] and the lack of backscatter [10] have previously been analyzed. CAX and CAXE can be used to visualize and quantify the dose at depths along the CTV. CAXL represents dose distributions at the surface outside the CTV. For instance, CAXL can be used to evaluate the dose at the breast if the patient's flank is 
treated or to evaluate the dose to the eyes if the patient's cheek is treated. This can be done prior to generation of treatment plan; thus, a physician/physicist can establish a priori understanding of dose distributions for different clinical scenarios. Similarly, DS dose distribution can be used for evaluation of the dose to the brainstem, optic nerves, and other related structures prior to HDR-BT scalp treatment.

\section{Verification of the prediction model}

Verification of the prediction model was performed on the same cohort of 37 patients involved in the first part of this study. After categorizing all patients based on their CTV geometry, it was found that the overall accuracy of this model was $1.9 \%$ (SD $=3.2 \%$ ). An example of the validation was presented for CBCL of the abdomen

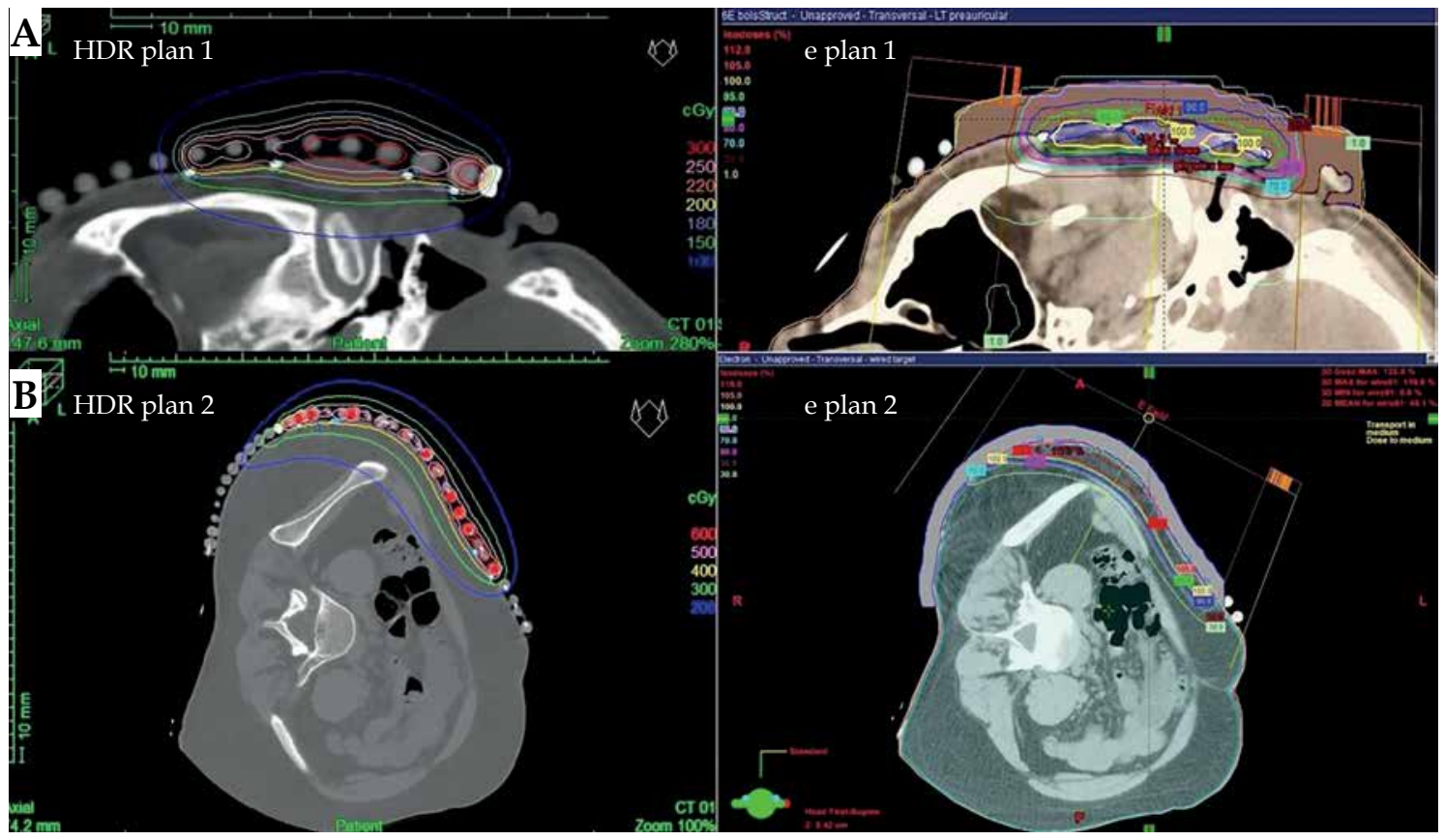

Fig. 4. Comparison between two EBRT and HDR-BT plans (representative cases): A) flat and small lesion, and B) curvy and large lesion

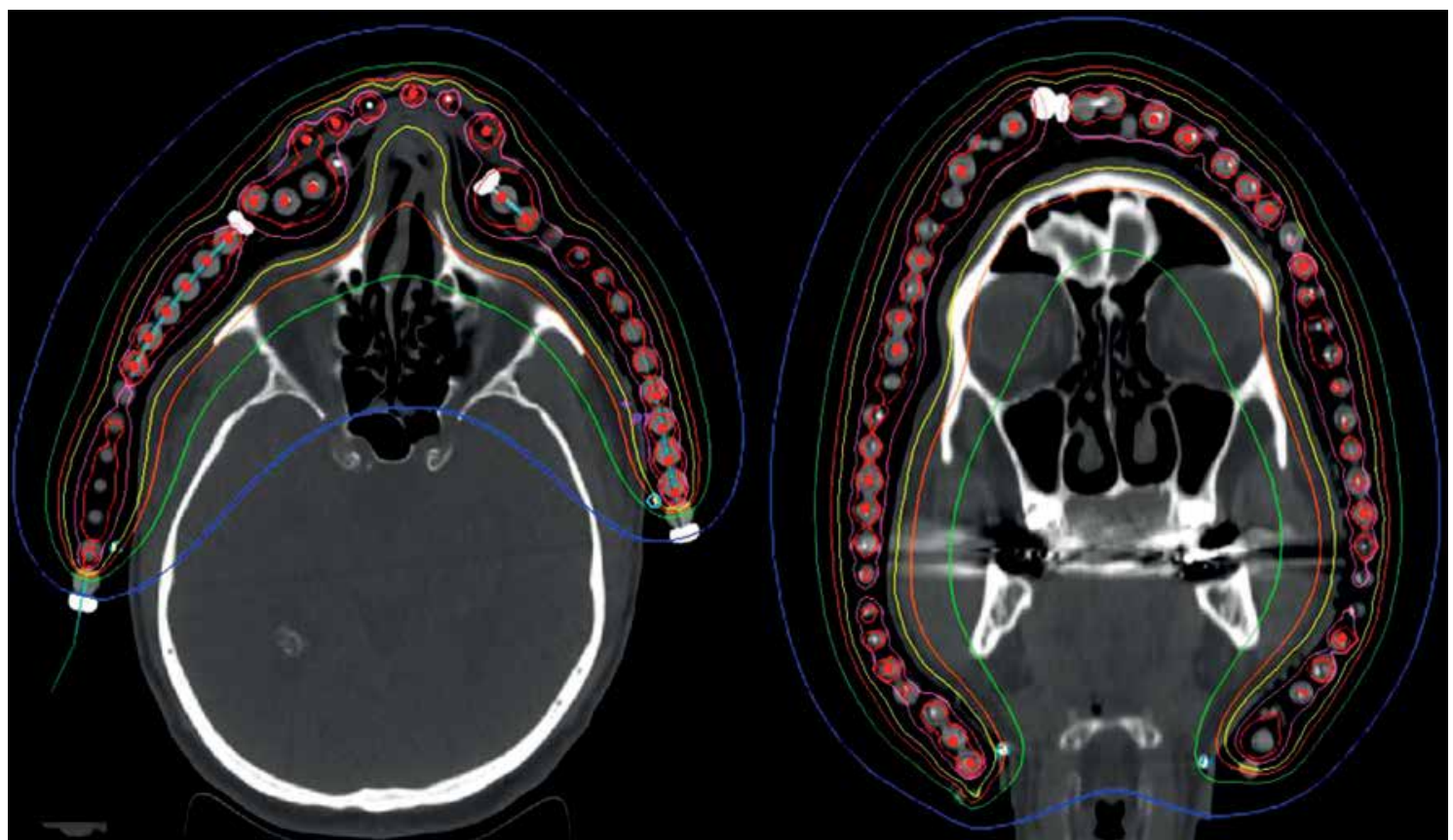

Fig. 5. Axial and coronal view of the whole face HDR-BT treatment plan. The $100 \%$ isodose line (yellow) was $3 \mathrm{~mm}$ under the skin. Treatment plans with EBRT, in general, give suboptimal results for such cases 
Table 2. Dose distributions in HDR surface brachytherapy. Depths were calculated from the source position (not from the skin)

\begin{tabular}{|c|c|c|c|c|c|c|}
\hline \multirow{2}{*}{$\begin{array}{l}\text { CTV size }\left(\mathrm{cm}^{2}\right) \\
10 \times 10\end{array}$} & \multicolumn{2}{|c|}{ CTV shape } & \multirow{2}{*}{$\begin{array}{c}5 \% \text { dose depth } \\
(\mathrm{mm})\end{array}$} & \multirow{2}{*}{$\begin{array}{c}50 \% \text { dose depth } \\
(\mathrm{mm})\end{array}$} & \multirow{2}{*}{$\begin{array}{c}90 \% \text { dose depth } \\
(\mathrm{mm})\end{array}$} & \multirow{2}{*}{$\begin{array}{c}125 \% \text { dose depth } \\
(\mathrm{mm})\end{array}$} \\
\hline & PLANE & CAX & & & & \\
\hline & & Edge & 130 & 21 & 9 & 6 \\
\hline & & Lateral & 96 & 14 & 7 & 5 \\
\hline \multirow[t]{3}{*}{$10 \times 10$} & CAX30 & CAX & 136 & 32 & 10 & 5 \\
\hline & & Edge & 110 & 16 & 8 & 5 \\
\hline & & Lateral & 92 & 16 & 8 & 5 \\
\hline \multirow[t]{3}{*}{$10 \times 10$} & CAX60 & CAX & 139 & 38 & 11 & 4 \\
\hline & & Edge & 104 & 15 & 6 & 4 \\
\hline & & Lateral & 98 & 16 & 7 & 5 \\
\hline \multirow[t]{3}{*}{$10 \times 10$} & $\mathrm{HCX}$ & CAX & 139 & 47 & 12 & 4 \\
\hline & & Edge & 99 & 14 & 6 & 4 \\
\hline & & Lateral & 100 & 17 & 6 & 4 \\
\hline \multirow[t]{3}{*}{$20 \times 20$} & PLANE & CAX & 151 & 34 & 11 & 5 \\
\hline & & Edge & 134 & 22 & 9 & 6 \\
\hline & & Lateral & 101 & 15 & 7 & 5 \\
\hline \multirow[t]{3}{*}{$20 \times 20$} & CAX30 & CAX & 164 & 39 & 11 & 4 \\
\hline & & Edge & 115 & 17 & 8 & 5 \\
\hline & & Lateral & 105 & 15 & 7 & 5 \\
\hline \multirow[t]{3}{*}{$20 \times 20$} & CAX60 & CAX & 192 & 52 & 12 & 5 \\
\hline & & Edge & 164 & 39 & 11 & 4 \\
\hline & & Lateral & 180 & 47 & 11 & 4 \\
\hline \multirow[t]{3}{*}{$20 \times 20$} & $\mathrm{HCX}$ & CAX & 192 & 52 & 12 & 5 \\
\hline & & Edge & 164 & 39 & 11 & 4 \\
\hline & & Lateral & 180 & 47 & 11 & 4 \\
\hline \multirow[t]{3}{*}{$\mathrm{d}=16 \mathrm{~cm}$} & DS & CAX & 194 & 61 & 12 & 4 \\
\hline & & Edge & 107 & 18 & 9 & 5 \\
\hline & & Lateral & 98 & 16 & 7 & 4 \\
\hline
\end{tabular}

(Figure S10A) and whole scalp treatment of angiosarcoma (Figure S10B). The accuracy of position of the isodose lines at the depths was $1.1 \mathrm{~mm}$ for the $50 \%$ isodose line, and $1.9 \mathrm{~mm}$ for the lower isodose lines. Figure S10 is presented in the Supplement section.

\section{Discussion}

In this study, we aimed to answer a common question in the treatment of skin malignancies (when resources are available for both options): should brachytherapy or electrons be used for a specific treatment? The study consists of two principal parts.

The first part was a dosimetric comparison between EBRT and HDR-BT of 37 representative cases with various CTV geometries. Since the prescription depth for treatments was $3 \mathrm{~mm}$, at least $1 \mathrm{~cm}$ bolus had to be used for $6 \mathrm{MeV}$ treatment plans. However, it was not always possible to maintain constant depth along the target with EBRT. This effect became pronounced when the CTV started curving for $30^{\circ}$ or more with respect to the CAX (the CAX distance in the axial slices became greater than $20 \mathrm{~mm}$ ). In such cases, a decrease of CTV coverage $\left(D_{90}\right.$ and $\left.D_{95}\right)$ up to $40 \%$ was observed. Therefore, it was confirmed that the curvature of the target played a crucial role in such treatments. As the curvature increased, the degradation of EBRT plans also increased. As a result, HDR-BT showed superior results in these cases since this modality could 'curve' the dose and adapt to the target. In these cases, the matched-field EBRT might be a better plan option than single-field EBRT. However, this would raise a well-known concern about electron field matching [11]. The degradation of $D_{90}$ and $D_{95}$ for EBRT plans stayed constant as the curvature increased; however, this finding did not have relevant clinical implications. The trend of decreasing $D_{90}, D_{95}$, and $D_{100}$ was found to be similar between HDR-BT and EBRT. Hot-spots and highdose regions inside the CTV (evaluated through D0.1 $\mathrm{cm}^{3}$, $\mathrm{D} 0.5 \mathrm{~cm}^{3}$, and $\mathrm{D} 2 \mathrm{~cm}^{3}$ ) were found to be within the same range (approximately of $\pm 7 \%$ ) for $\mathrm{D} 2 \mathrm{~cm}^{3}$. However, the EBRT plans showed better homogeneity than the HDRBT plans, since the D0.1 $\mathrm{cm}^{3}$ and D0.5 $\mathrm{cm}^{3}$ were lower, approximately $4 \%$ and up to $12 \%$, respectively. Another dosimetric advantage of the EBRT plans was lower skin 
dose in the range of $6-20 \%$. Similarly, the irradiated volume for the EBRT plans was lower, up to $50 \%$. Therefore, this indicates that the EBRT plans resulted in comparable or even better dosimetry when the target was flat. The above dosimetric benefits were still noted when the CTV started curving above $15-20^{\circ}$; however, their relevance was low in these situations, since the coverage of CTVs became insufficient for the treatment. When the size of CTV started increasing, a similar trend was observed. It was noted that the HDR-BT plans were more suitable for clinical use when the CTV became large enough; often, it was necessary to generate matched-beam EBRT plans. However, it was noticed that curvature of the CTV was a more important factor in modality selection than the size of CTV.

Once the initial choice between EBRT and HDR-BT was made based on target geometry, the question remains what HDR-BT dosimetry for complicated topology targets can be achieved. When dose distributions for a standard CTV of $10 \times 10 \mathrm{~cm}^{2}$ and various curvatures (PLANE, CAX30, CAX60, and HCX) were analyzed, a strong similarity in dose distributions along the CAX was noted for $5 \%$ isodose lines and below (at a depth of $140 \mathrm{~mm}$ ) as well as for isodose lines, ranging between 95 and $200 \%$. The most prominent differences were revealed for isodose lines of a) 50\%, ranging between 27 and $47 \mathrm{~mm}$ for PLANE and HCX, respectively, and b) $70 \%$ ranging between 16.5 and $39 \mathrm{~mm}$ for PLANE and HCX, respectively. These isodose lines for CAX30 and CAX60 were within the same range. The $5 \%$ isodose line for the DS custom-made applicator reached $194 \mathrm{~mm}$ along the CAX, whereas isodose lines above $70 \%$ were comparable with the one in HCX. The CAXE distribution was found to have a smaller range than the one presented along the CAX. For example, the range of depths was greatest at $25 \%$ isodose line $(44 \mathrm{~mm}$ for PLANE and $3 \mathrm{~mm}$ for HCX). In this case, differences in depth were noted for all high isodose lines ( $90 \%$ and above), except for the CAX distributions. A strong similarity in dose distribution was noted outside the field (CAXL) for isodose lines of $50 \%$ and greater. For example, the $25 \%$ isodose line was noticed at a depth of $29.5 \mathrm{~mm}$ for PLANE and $35 \mathrm{~mm}$ for HCX. As the CTV increased $\left(20 \times 20 \mathrm{~cm}^{2}\right)$, the range of lower isodose lines increased along the central axes (151 mm for PLANE, and $192 \mathrm{~mm}$ for HCX), maintaining a high range even close to the prescription depth (for $90 \%$ isodose lines, the difference in depth was $1.2 \mathrm{~mm}$ for given applicators). There were similar trends for the CAXL dose distribution of $20 \times 20 \mathrm{~cm}^{2}$ applicator, but not for $10 \times 10 \mathrm{~cm}^{2}$ one. Apart from some site-specific studies, such comparisons have not yet been reported. Some partial findings and initial results of this study had been reported previously [12].

\section{Conclusions}

In this article, the selection criteria between two radiation modalities (EBRT and HDR-BT) used in the treatments of surface targets were presented. The general conclusion was that both techniques could be successfully used in the treatments of superficial targets. The quality of treatment for each technique and the outcome were dependent on the target geometry, i.e., size, curvature, and topology. The decrease in quality of electron plans was found to be more dependent on target curvature than on CTV size. It was shown that HDR-BT provided superior CTV coverage in comparison with EBRT when the surface was very large, complex, curvy, or rounded (arm, leg, flank), and when the topology was complicated (fingers, whole face). A notable advantage of HDR-BT plans was the ability to deform and adjust dose over large irregular surfaces using commercial flap applicators or custom-made applicators. However, for flat, smaller, and medium-sized targets (with an area of less than $12 \times 12 \mathrm{~cm}^{2}$ ), EBRT yielded comparable plans and improved dose homogeneity. A predictive model using dose distributions was developed for improved understanding of isodose line distributions for various clinical scenarios to assist in deciding on which modality to deploy.

\section{Disclosure}

The authors report no conflict of interest.

\section{References}

1. Fletcher GH. Introduction. In: Tapley N (Ed.). Clinical applications of the electron beam. John Wiley \& Sons, New York, NY 1976; 1.

2. Khan FM, Gibbons JP. Chapter 14: Electron beam therapy. In: Khan's the physics of radiation therapy. Lippincott Williams \& Wilkins, Philadelphia 2014.

3. Khan FM, Doppke KP, Hogstrom KR et al. Clinical electron-beam dosimetry: Report of AAPM Radiation Therapy Committee Task Group No. 25. Med Phys 1991; 18: 73-109.

4. Gerbi BJ, Antolak JA, Deibel FC et al. Recommendations for clinical electron beam dosimetry: Supplement to the recommendations of Task Group 25. Med Phys 2009; 36: 3239-3279.

5. Gibbons JP, Antolak JA, Followill DS et al. Monitor unit calculations for external photon and electron beams: Report of the AAPM Therapy Physics Committee Task Group No. 71. Med Phys 2014; 41: 031501.

6. Likhacheva AO, Devlin PM, Buzurovic IM. Chapter 8: Skin brachytherapy. In: Brachytherapy applications and techniques. Demos Medical Publishing, New York 2015; 211-234.

7. Devlin PM, Gaspar LE, Buzurovic I et al. American College of Radiology-American Brachytherapy Society practice parameter for electronically generated low-energy radiation sources. Brachytherapy 2017; 16: 1083-1090.

8. Likhacheva A, Awan M, Barker CA et al. Definitive and postoperative radiation therapy for basal and squamous cell cancers of the skin: executive summary of an American Society for Radiation Oncology Clinical Practice Guideline. Pract Radiat Oncol 2020; 10: 8-20.

9. Bhagwat M, Buzurovic I, O'Farrell D et al. Effect of heterogeneity on dose deposited by a flat HDR surface applicator. Brachytherapy 2014; 13: S98-S99.

10. Aldelaijan S, Bekerat H, Buzurovic I et al. Dose comparison between TG-43-based calculations and radiochromic film measurements of the Freiburg flap applicator used for high-dose-rate brachytherapy treatments of skin lesions. Brachytherapy 2017; 16: 1065-1072.

11. Lachance B, Tremblay D, Pouliot J. A new penumbra generator for electron fields matching. Med Phys 1997; 24: 485-495.

12. Buzurovic I, Hansen J, Bhagwat $\mathrm{M}$ et al. Characterization of dose distributions in high-dose-rate surface brachytherapy. Med Phys 2015; 42: 3369-3370. 


\section{Supplement}

This section contains Figures S1-S9 for various applicator shapes and sizes, as in Figures 1 and 2, so the proposed prediction model of dose distribution can be used in clinical scenarios. Figures S1-S9 were organized to: a) show the isodose distributions at greater depths (> $100 \mathrm{~mm}$ ), while b) representing the same dose distribution at shallow depths $(<20 \mathrm{~mm})$. Hence, it is possible to present doses to the deep-seated structures as well as doses close to the surface and prescription depths. In this model, the prescription dose $(100 \%)$ is at $8 \mathrm{~mm}$ from the source plane, which is equivalent to the common clinical scenario, in which the prescription depth is $3 \mathrm{~mm}$ under the skin (to reach the dermis). Therefore, all depths given in Figures S1-S9 are depths from the source position and not from the skin. Figure S10 is a representative case used for validation of the prediction model.
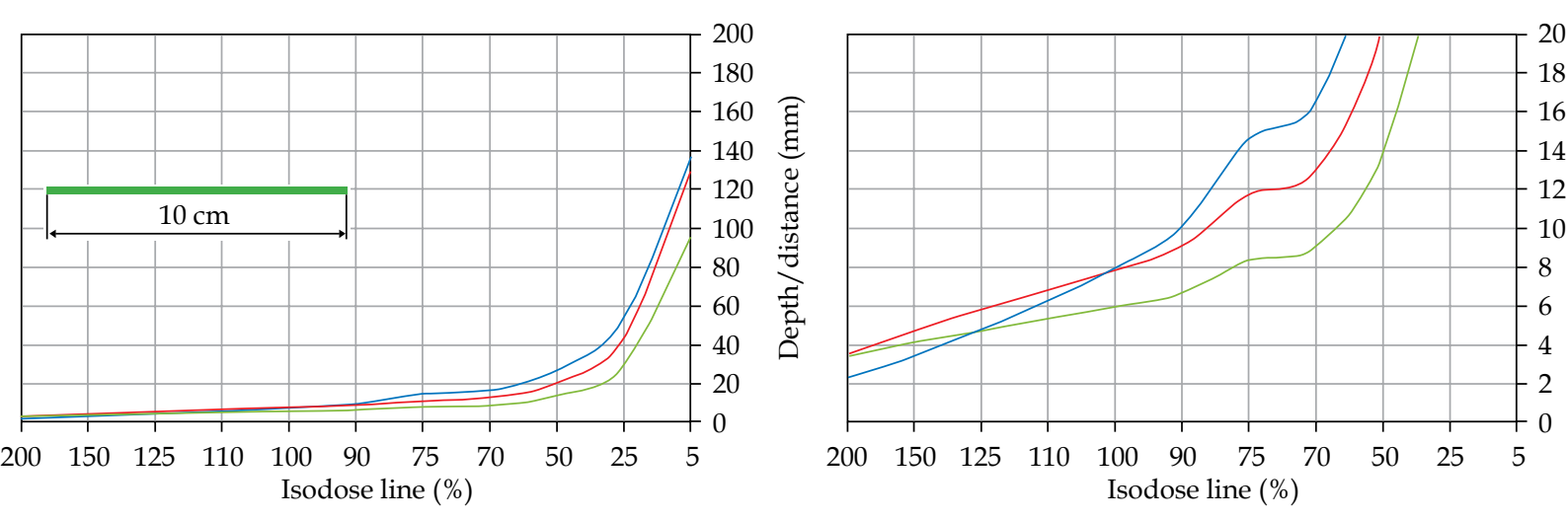

Fig. S1. CAX, CAXE, and CAXL dose distributions for PLANE at a greater depth (left) and a shallow depth (right) and CTV of $10 \times 10 \mathrm{~cm}^{2}$
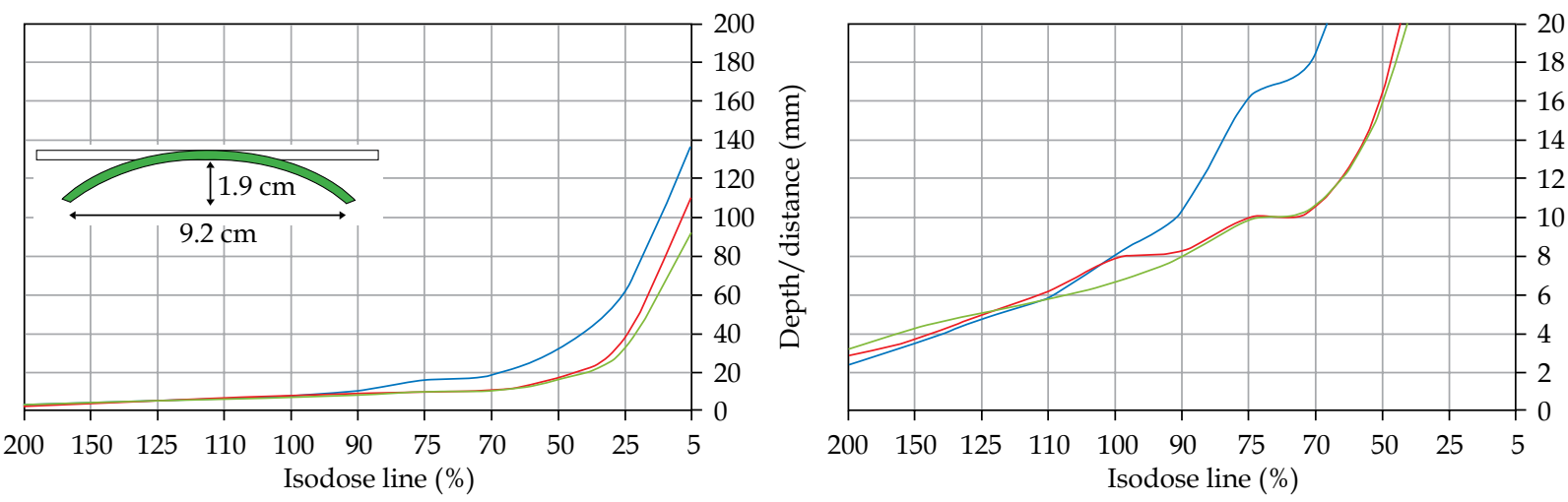

Fig. S2. CAX, CAXE, and CAXL dose distribution for CAX30 at a greater depth (left) and a shallow depth (right) and CTV of $10 \times 10 \mathrm{~cm}^{2}$
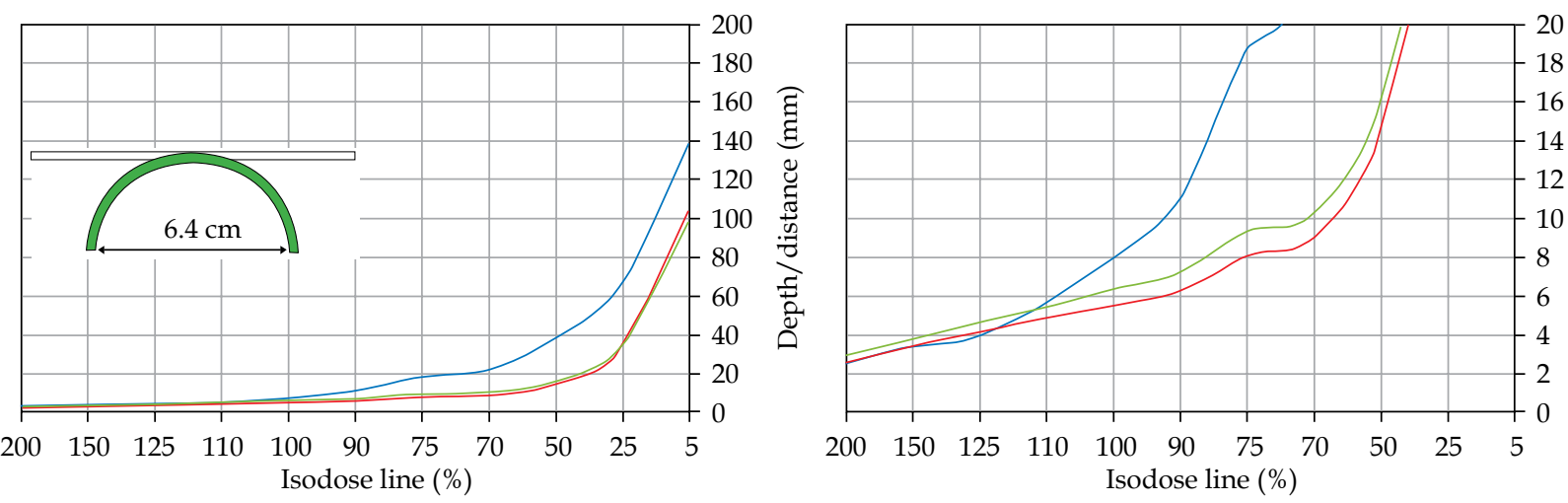

Fig. S3. CAX, CAXE, and CAXL dose distribution for CA60X at a greater depth (left) and a shallow depth (right) and CTV of $10 \times 10 \mathrm{~cm}^{2}$ 

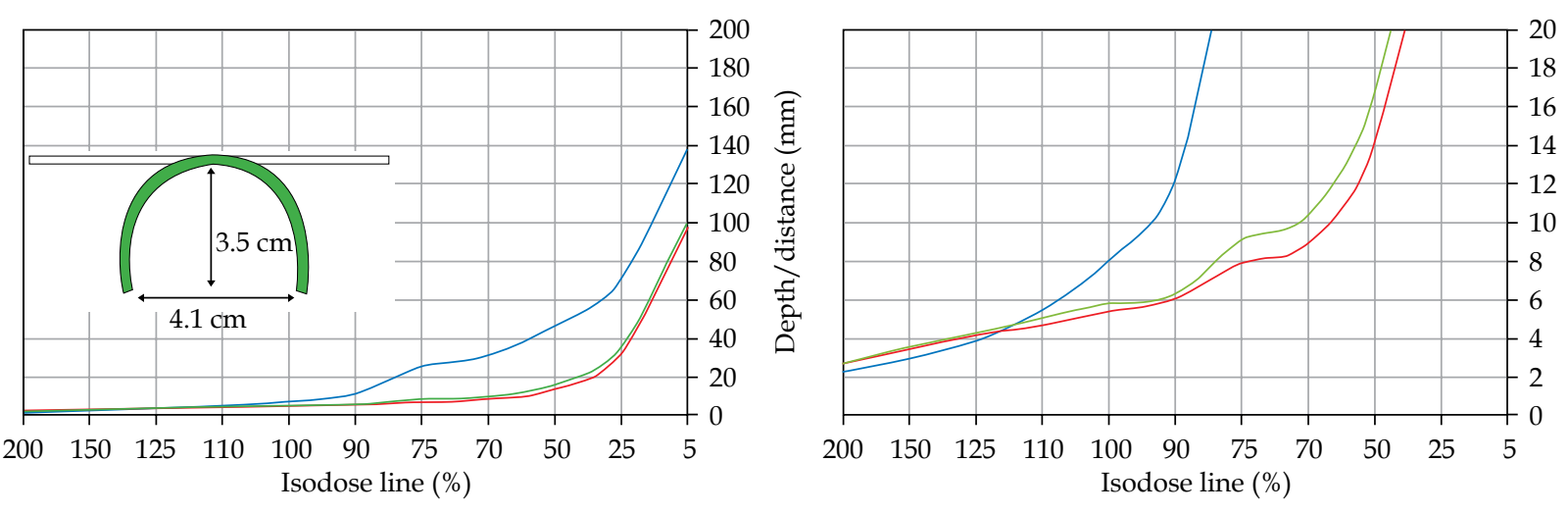

Fig. S4. CAX, CAXE, and CAXL dose distribution for HCX at a greater depth (left) and a shallow depth (right) and CTV of $10 \times 10 \mathrm{~cm}^{2}$
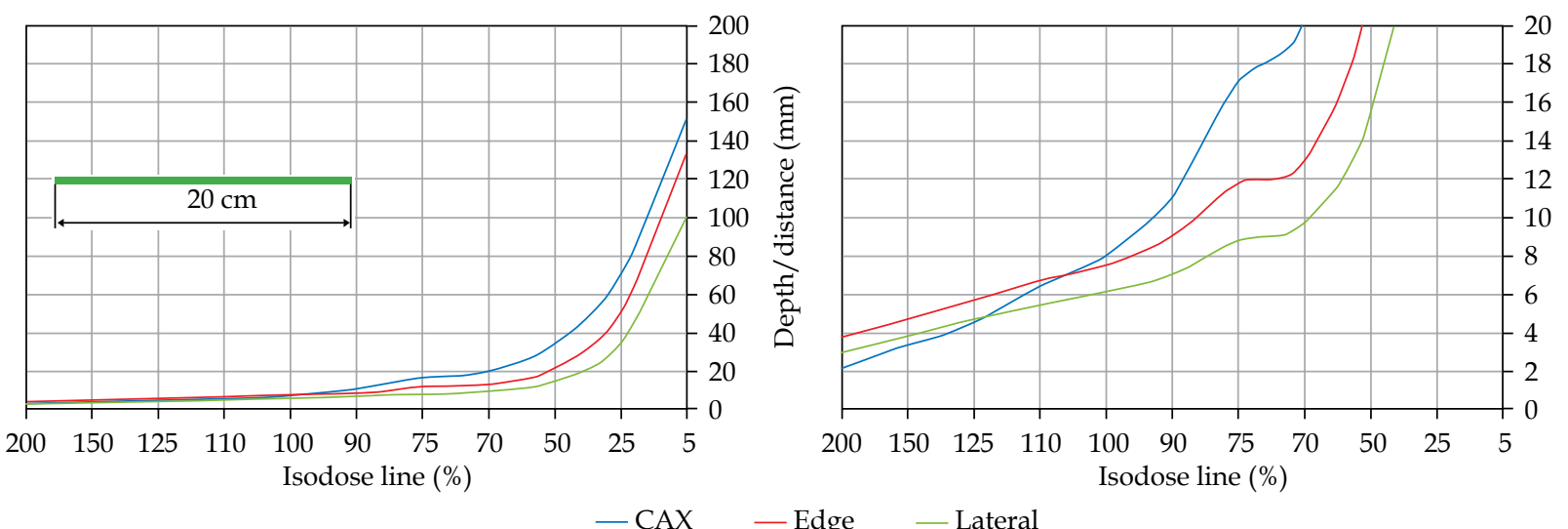

Fig. S5. CAX, CAXE, and CAXL dose distribution for PLANE at a greater depth (left) and a shallow depth (right) and CTV of $20 \times 20 \mathrm{~cm}^{2}$
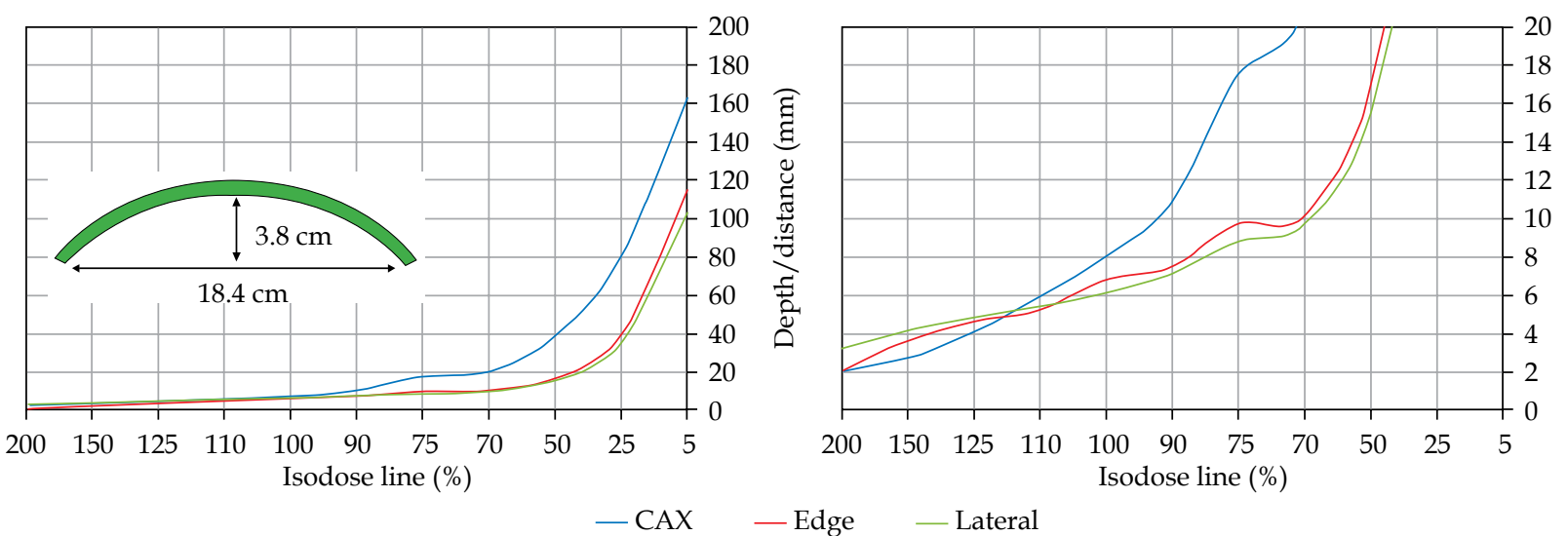

Fig. S6. CAX, CAXE, and CAXL dose distribution for CAX30 at a greater depth (left) and a shallow depth (right) and CTV of $20 \times 20 \mathrm{~cm}^{2}$ 

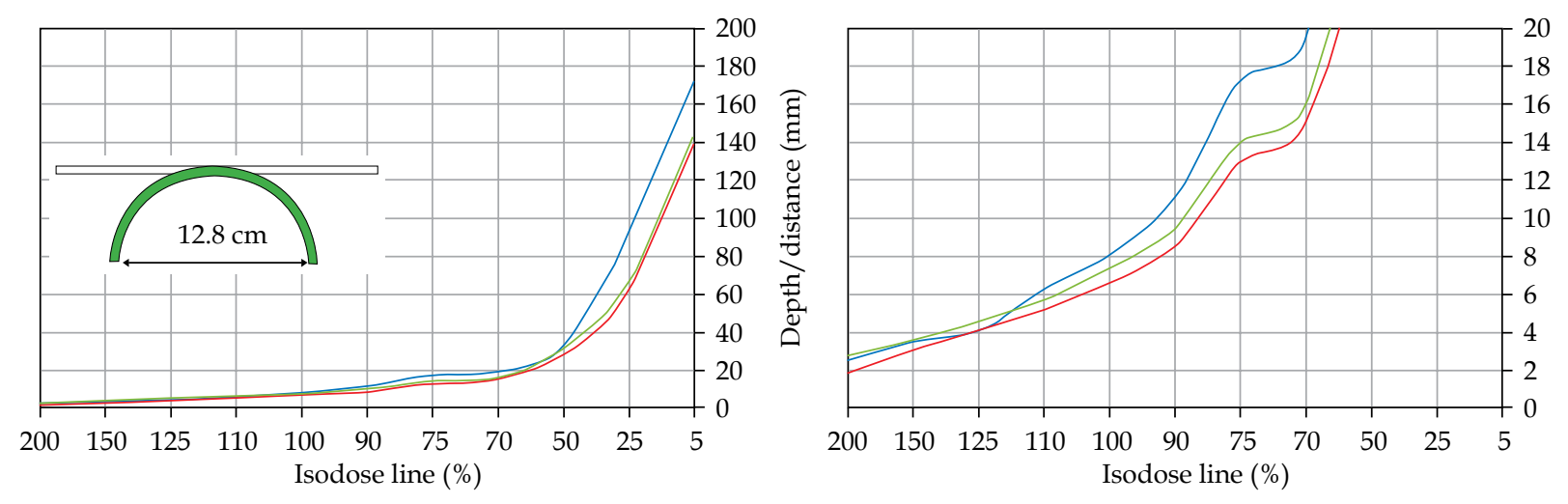

- CAX — Edge — Lateral

Fig. S7. CAX, CAXE, and CAXL dose distribution for CAX60 at a greater depth (left) and a shallow depth (right) and CTV of $20 \times 20 \mathrm{~cm}^{2}$
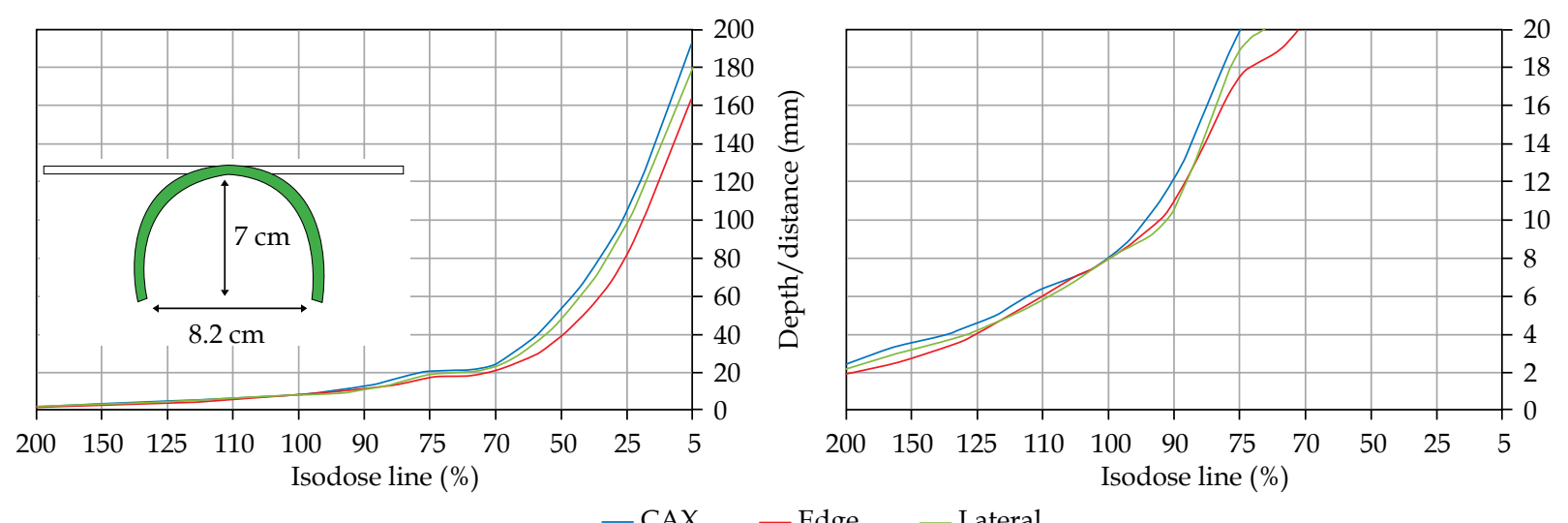

Fig. S8. CAX, CAXE, and CAXL dose distribution for HCX at a greater depth (left) and a shallow depth (right) and CTV of 20 $\times 20 \mathrm{~cm}^{2}$
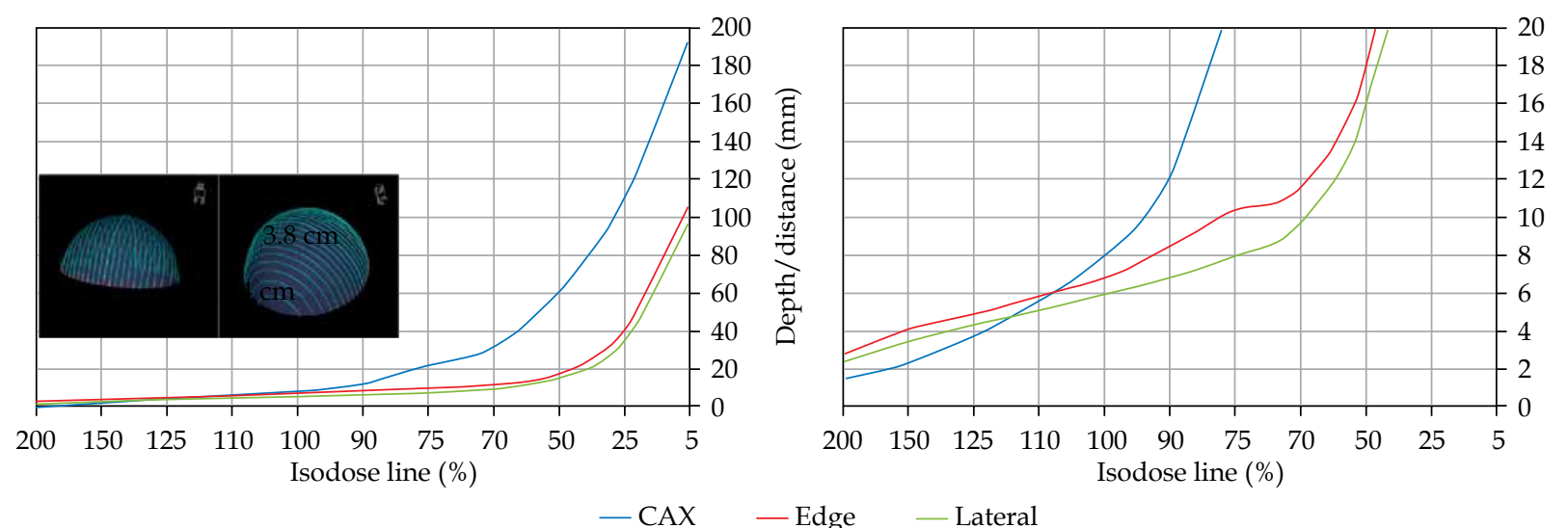

Fig. S9. CAX, CAXE, and CAXL dose distribution for DS at a greater depth (left) and a shallow depth (right)

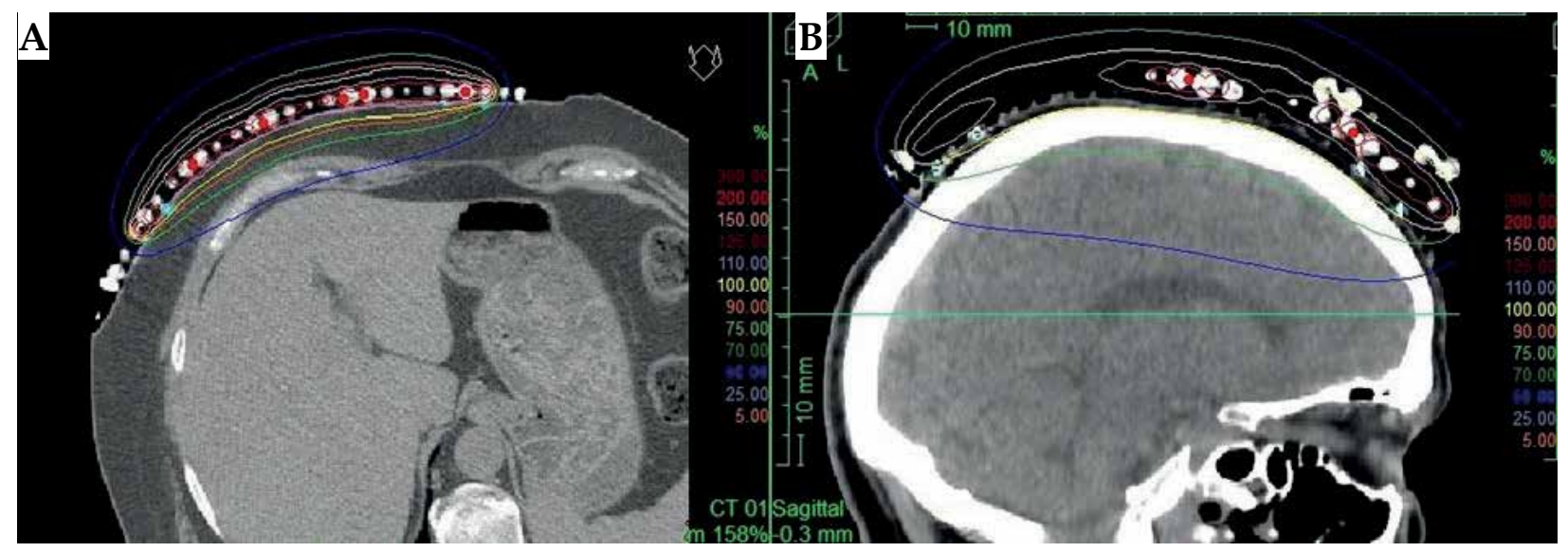

Fig. S10. Verification of dose distribution for A) $20 \times 23 \mathrm{~cm}^{2}$ treatment of the abdomen, and B) partial scalp treatment 